\title{
Molecular mechanisms of luteinization
}

\author{
Norihiro Sugino \\ Department of Obstetrics and Gynecology, Yamaguchi University Graduate School of Medicine, Ube, Japan
}

Dynamic changes in steroidogenesis occur in ovarian granulosa cells during ovulation after the LH surge. The ovulatory LH surge induces rapid up-regulation of steroidogenic acute regulatory (StAR) protein and rapid down-regulation of aromatase (Cyp 19a1) in granulosa cells undergoing luteinization during ovulation. These rapid changes in StAR and Cyp19a1 gene expression after the LH surge efficiently facilitate progesterone production, which plays a crucial role in ovulation and the following luteinization. Recently, it has become clear that epigenetic regulation such as histone modifications and DNA methylation play a key role in gene expression through the chromatin remodeling of the promoter region. This study reports the in vivo evidence that epigenetic mechanisms including histone modifications, DNA methylation and chromatin remodeling are involved in the rapid changes of StAR and Cyp 19a 1 gene expression in granulosa cells undergoing luteinization during ovulation.

Keywords: Cyp19a1; Epigenetics; Histone modification; Luteinization; StAR

\section{Introduction}

Dynamic changes in steroidogenesis occur in ovarian granulosa cells undergoing luteinization during ovulation after the LH surge. The ovulatory LH surge interacts with LH receptor and stimulates c-AMP mediated signal transduction pathway including epidermal growth factor-like factors (amphiregulin and epiregulin) and subsequent ERK-1 and -2 signals, and thereafter induces rapid up-regulation of steroidogenic acute regulatory $(S t A R)$ protein, a rate-limiting enzyme for progesterone synthesis [1], while the expression of aromatase (Cyp19a1), a key enzyme for estrogen synthesis, is rapidly suppressed after the LH surge [2]. These rapid changes in StAR and Cyp19a1 gene expression after the LH surge efficiently facilitate progesterone production, which plays a crucial role in ovulation and the following luteinization. The biological effect of progesterone via progesterone receptors is necessary for ovulation. Suppression of progesterone synthesis by $3 b$-hydroxysteroid-dehydrogenase inhibitors blocks ovulation in rats [3]. Progesterone receptornull mice fail to ovulate due to the impaired follicle rupture [4]. However, it is unclear about molecular mechanisms for the rapid changes in StAR and Cyp19a 1 gene expression in granulosa cells undergoing luteinization after the ovulatory LH surge.

The regulation of gene expression by transcription factors has been well accepted. Recently, it has become clear that epigenetic mechanisms such as histone modifications and DNA methylation play a key role in transcription through the chromatin remodeling of the promoter region. We have reported that histone modifications are involved in the regulation of gene expression of the human endometrium [5-8], and DNA methylation is involved in the pathogenesis of certain reproductive diseases such as endometriosis and uterine leiomyomas [9-14]. This study reports the in vivo evidence that epigenetic mechanisms including histone modifications, DNA methylation and chromatin remodeling are involved in the rapid changes of StAR and Cyp19a1 gene expression in granulosa cells undergoing luteinization during ovulation [15].

Received: 2013.11.22. Revised: 2014.1.29. Accepted: 2014.2.4.

Corresponding author: Norihiro Sugino

Department of Obstetrics and Gynecology, Yamaguchi University Graduate School of Medicine, Minamikogushi 1-1-1,

Ube 755-8505, Japan

Tel: +81-836-22-2286 Fax: +81-836-22-2287

E-mail: sugino@yamaguchi-u.ac.jp

Articles published in Obstet Gynecol Sci are open-access, distributed under the terms of the Creative Commons Attribution Non-Commercial License (http://creativecommons. org/licenses/by-nc/3.0/) which permits unrestricted non-commercial use, distribution, and reproduction in any medium, provided the original work is properly cited.

Copyright $\odot 2014$ Korean Society of Obstetrics and Gynecology 


\section{Obstetrics \& Gynecology Science}

Vol. 57, No. 2, 2014

\section{Gene expressions of StAR and Cyp19a1}

The present study was reviewed and approved by the Committee for the Ethics on Animal Experiment in Yamaguchi University Graduate School of Medicine. Female Sprague-Dawley rats (21-24 days old) were injected subcutaneously with 15 IU of equine chorionic gonadotropin (eCG) followed by $15 \mathrm{IU}$ of human chorionic gonadotropin (hCG) 48 hours after eCG injection to induce ovulation and luteinization. Granulosa cells were isolated before $\mathrm{hCG}$ (0 hours), and 4, 8, and 12 hours after hCG injection. StAR mRNA levels rapidly increased after hCG injection, reached a peak at 4 hours, and remained high until 12 hours after hCG injection (Fig. 1). In contrast, Cyp19a1 mRNA levels gradually decreased after hCG injection and were significantly lower at 8 and 12 hours compared with 0 hours (Fig. 1).

\section{Regulation of gene expression by epigenetic mechanisms}

In addition to transcription factors, epigenetic mechanisms such as DNA methylation and histone modifications are involved in transcriptional regulation. DNA methylation occurs at cytosines within $\mathrm{CpG}$ dinucleotides, which can silence gene expression by altering chromatin structure and preventing the binding of transcription factors $[16,17]$. Histone modifications also affect chromatin structure. Chro- matin structure of the promoter is critical for the interaction of transcription factors with response elements $[18,19]$. Histone modifications, such as acetylation of histone $\mathrm{H} 3$ and histone $\mathrm{H} 4$ or trimethylation of the site of lysine 4 on histone $\mathrm{H3}$ (H3K4me3) activate transcription by loosening the chromatin structure and allowing the recruitment of transcription factors to their response elements. On the other hand, histone modifications such as trimethylation of the site of lysine 9 and 27 on histone H3 (H3K9me3 and H3K27me3) inactivate transcription by causing the chromatin to become more condensed [19].

\section{DNA methylation status of the StAR and Cyp19a1 promoters}

The DNA methylation status at the CpG sites in the StAR promoter region $(-1,722$ to $-13 \mathrm{bp})$ was analyzed by sodium bisulfite genomic sequencing (Fig. 2A). The promoter region until -500 bp is especially important for transcription because a number of binding sites of various transcription factors are located in this region. There are $6 \mathrm{CpG}$ sites in the proximal promoter region. Before hCG injection (0 hours), the $\mathrm{CpG}$ sites in the proximal region were unmethylated, whereas the $C p G$ sites in the distal region were methylated (Fig. 2A). These methylation statuses did not change at any time observed after hCG injection, suggesting that the

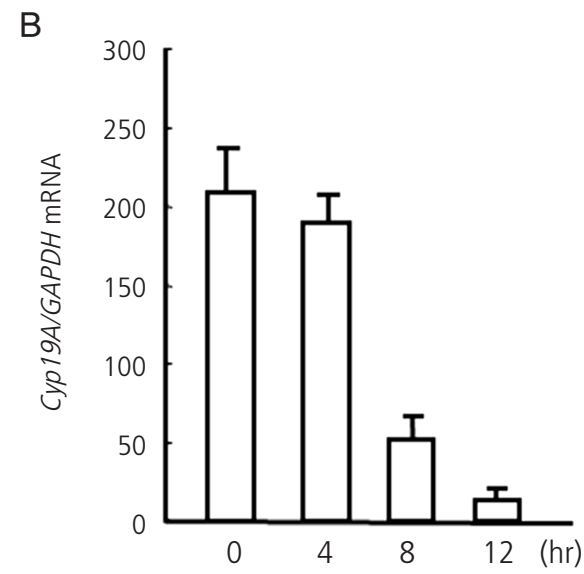

Fig. 1. Changes in mRNA expression of $\operatorname{StAR}(\mathrm{A})$ and Cyp19a1 (B) in granulosa cells undergoing luteinization during ovulation. Granulosa cells were obtained from rats treated with equine chorionic gonadotropin before ( 0 hours) and 4,8 , and 12 hours after human chorionic gonadotropin injection. mRNA levels were measured by real-time reverse transcription polymerase chain reaction. Data were expressed as a ratio of StAR/Cyp19a1 mRNA to GAPDH (internal control). Each bar represents the mean \pm SEM of five animals. The data were reported in the reference no. 15. 


\section{Obstetrics \& Gynecology Science}

Norihiro Sugino. Molecular mechanisms of luteinization

A

StAR promoter

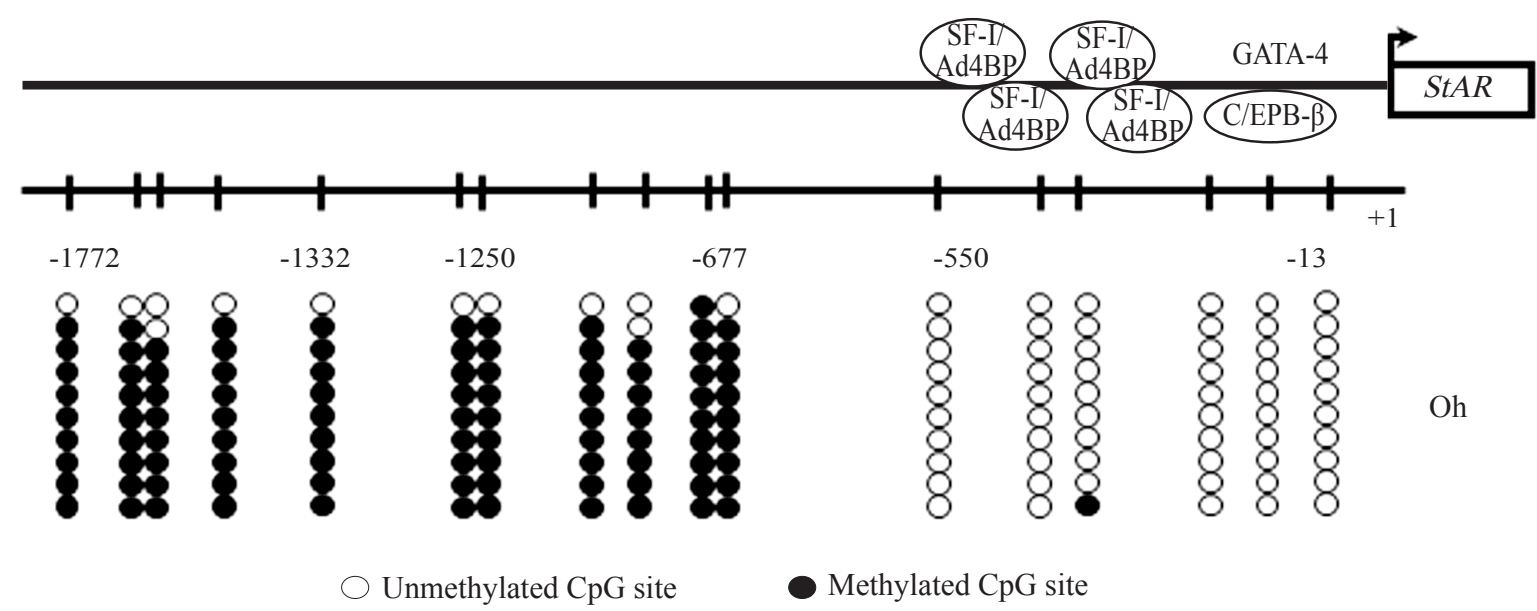

B

Cyp19al promoter II

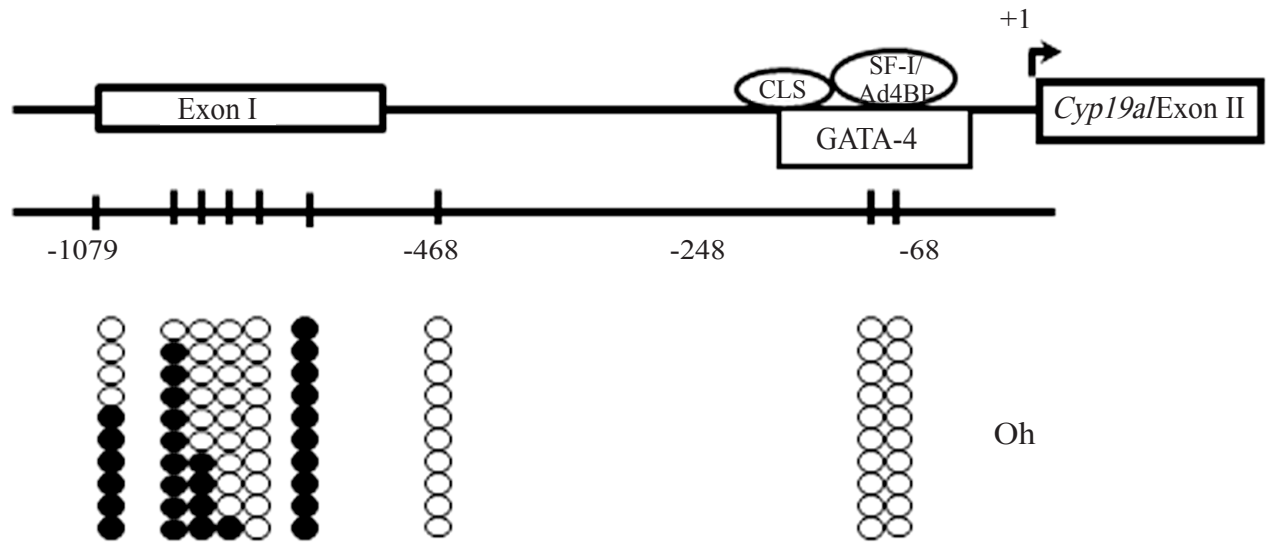

Fig. 2. DNA methylation statuses of the promoters of StAR (A) and Cyp19a 1 (B) in granulosa cells undergoing luteinization during ovulation. The diagram shows the distribution of $\mathrm{CpG}$ sites and location of the response elements for transcriptional factors, in which the tick marks indicate the positions of $\mathrm{CpG}$ sites. The position of the transcription start site is designated as +1 . DNA methylation statuses of the CpG sites were analyzed by sodium bisulfite genomic sequencing in the StAR promoter region (-13 to -1772 bp) and Cyp 19a 1 promoter region (-68 to $-1079 \mathrm{bp}$ ). Granulosa cells were obtained from rats treated with equine chorionic gonadotropin before (0 hours) and 4 hours, and 12 hours after human chorionic gonadotropin (hCG) injection. The data before hCG (0 hours) were only shown because the methylation statuses did not change at any time observed after hCG injection. Open and filled circles indicate unmethylated and methylated CPG status, respectively. The data were reported in the reference no. 15. SF-1/Ad4BP, steroidogenic factor $1 /$ adrenal 4 binding protein; GATA-4, GATA binding protein 4; C/EBP $\beta$, CCAAT/enhancer binding protein $\beta$; CLS, cAMP-responsive element-like sequence.

proximal region of the StAR promoter between $-550 \mathrm{bp}$ and $-13 \mathrm{bp}$ is an important region for transcription.

The Cyp19a1 promoter II is located upstream of exon II, and is recognized as a tissue-specific promoter region in the rat ovary $[20,21]$. The region until $-150 \mathrm{bp}$ is critical for transcription. There are 2 CG sites in the promoter between - 248 and -68 bp. Both CpG sites were unmethylated before (0 hours) hCG injection (Fig. 2B) and did not change after hCG injec- tion, suggesting that this region is important for transcription.

\section{Histone modifications of the StAR and Cyp19a1 promoters}

The change in histone modifications of the StAR promoter region after hCG injection was analyzed by chromatin im- 


\section{Obstetrics \& Gynecology Science}

Vol. 57, No. 2, 2014

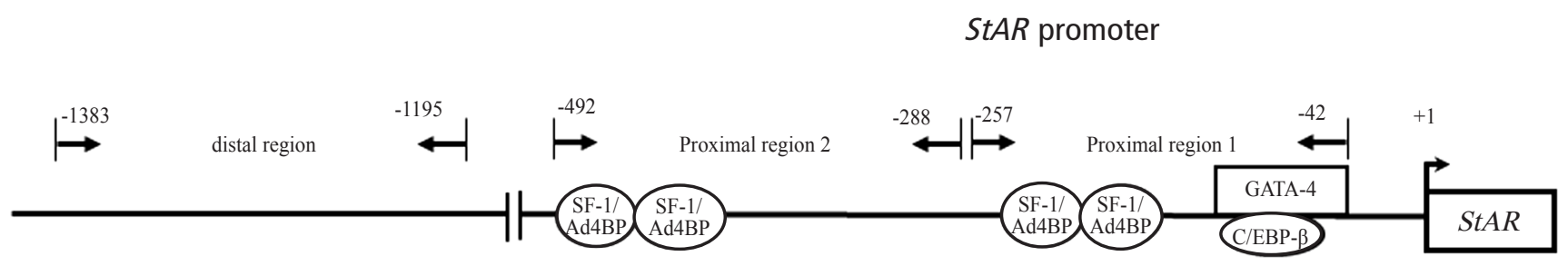

Cyp19al promoter II

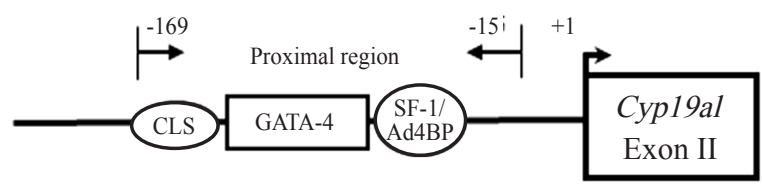

Fig. 3. Locations of the response elements for transcriptional factors in the rat StAR promoter region and Cyp19a1 promoter region. Analyzed regions of StAR (proximal region 1, proximal region 2, and distal region) and of Cyp19a1 (proximal region) for histone modifications by chromatin immunoprecipitation assay are shown. SF-1/Ad4BP, steroidogenic factor 1/adrenal 4 binding protein; GATA-4, GATA binding protein 4; C/EBP $\beta$, CCAAT/enhancer binding protein $\beta$; CLS, CAMP-responsive element-like sequence.

munoprecipitation (ChIP) assay in the proximal region (proximal region 1, -257 to $-42 \mathrm{bp}$; proximal region 2, -492 to $-288 \mathrm{bp}$ ) and the distal region (-1383 to $-1195 \mathrm{bp}$ ) (Fig. 3). The $5^{\prime}$-upstream region between -257 and -42 bp of the $S t A R$ gene (proximal region 1 ) is especially critical for the regulation of StAR gene expression. Since the change in histone modifications in the proximal region 2 was similar to those in the proximal region 1 , the result of the proximal region 1 was only shown in Fig. 4 . Active marks of histone modifications such as histone-H4 acetylation and H3K4me3 increased after hCG injection, whereas repressive marks such as H3K9me3 and H3K27me3 decreased after hCG injection (Fig. 4). Histone-H3 acetylation did not change after hCG injection (data not shown). On the other hand, in the distal region, active histone modification marks did not change after hCG (data not shown).

The change in histone modifications of the Cyp19a1 promoter II after hCG injection was analyzed by ChIP assay in the region between -169 and -15 bp (Fig. 3). Active marks of histone modifications such as histone-H4 acetylation and $\mathrm{H} 3 \mathrm{~K} 4 \mathrm{me} 3$ decreased after hCG injection, whereas repressive marks such as H3K27me3 increased after hCG injection (Fig. 4). HistoneH3 acetylation also decreased after hCG injection in a manner similar to histone-H4 acetylation (data not shown).

It is unclear how the promoter region-specific histone modifications are regulated. Further studies on the recruitment of histone modification enzymes such as histone acetyltrans- ferases, histone deacetylases, histone methyltransferases, or histone demethylases to each promoter region are needed.

\section{Chromatin structure in the StAR and Cyp19a1 promoters}

To investigate whether the change in histone modifications in the StAR and Cyp19a1 promoter regions actually alters the chromatin structure of the promoters, DNase I accessibility assay was performed. In the proximal region 1 of the StAR, DNase accessibility levels decreased after hCG injection, suggesting that the chromatin structure of the StAR promoter loosens after $\mathrm{hCG}$ injection (Fig. 5A). The change in chromatin structure of the proximal region 2 was similar to that in the proximal region 1 . On the other hand, DNase accessibility levels in the Cyp19a 1 promoter increased after hCG injection, suggesting that chromatin structure of the Cyp 19a 1 promoter becomes condensed after hCG injection (Fig. 5B).

\section{Binding activities of C/EBP $\beta$ and phosphorylated CREB to the StAR and Cyp19a1 promoters}

Recent studies using knockout mice or microarray analyses have shown that CAATT/enhancer-binding protein (C/EBP) 


\section{Obstetrics \& Gynecology Science}

Norihiro Sugino. Molecular mechanisms of luteinization

StAR promoter

proximal region 1

$\mathrm{AcH} 4$
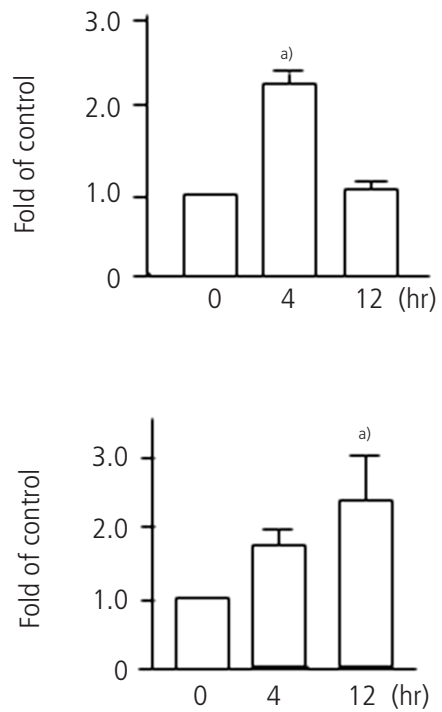

H3K9me3

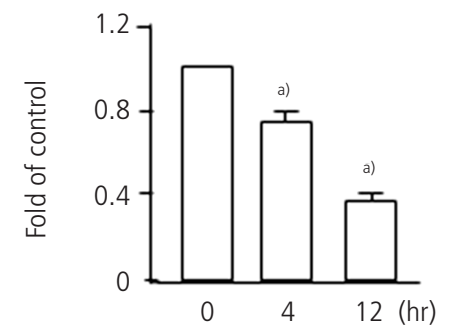

H3K27me3

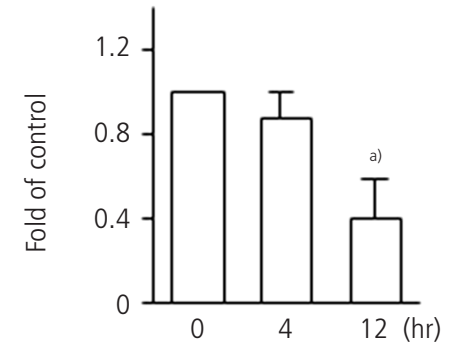

Cyp19al promoter
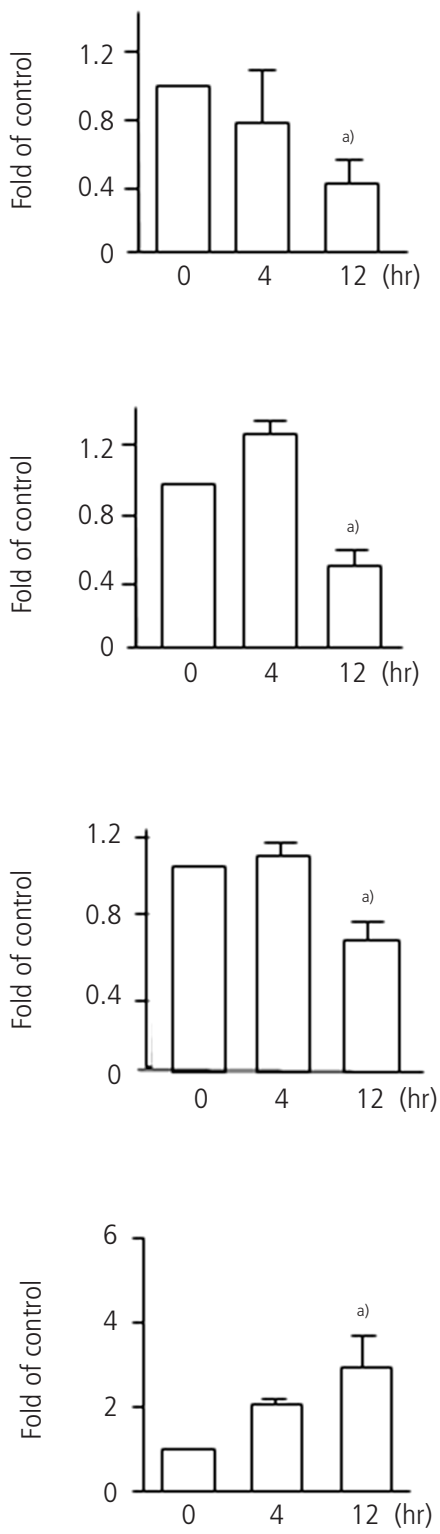

Fig. 4. Histone modifications of the promoters of StAR and Cyp19a1 in granulosa cells undergoing luteinization during ovulation. Granulosa cells were obtained from rats treated with equine chorionic gonadotropin before (0 hours) and 4 hours, and 12 hours after human chorionic gonadotropin injection. For histone modifications, histone-H4 acetylation (AcH4), trimethyl histone H3K4 (H3K4me3), trimethyl histone H3K9 (H3K9me3) and trimethyl histone H3K27 (H3K27me3) were analyzed by chromatin immunoprecipitation assay in each region of the StAR promoter (proximal region 1, proximal region 2, and distal region) and the Cyp19a1 promoter (Fig. 3). The data of the proximal region-1 of the StAR promoter are shown. AcH4 and H3K4me3 activate transcription while H3K9me3 and H3K27me3 inactivate transcription. Data were expressed as a ratio of the value of 0 hours. Values are mean \pm SEM of more than three independent experiments. ${ }^{\text {a) }} P<0.05$ versus 0 hours. The data were reported in the reference no. 15. 


\title{
Obstetrics \& Gynecology Science
}

\author{
Vol. 57, No. 2, 2014
}

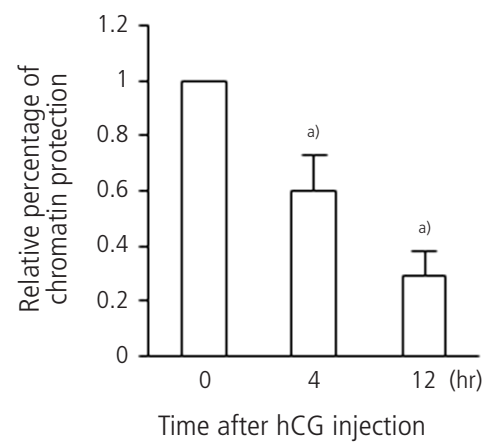

C

C/EBP $\beta$ binding activities in the StAR proximal promoter-1

$\lg G$
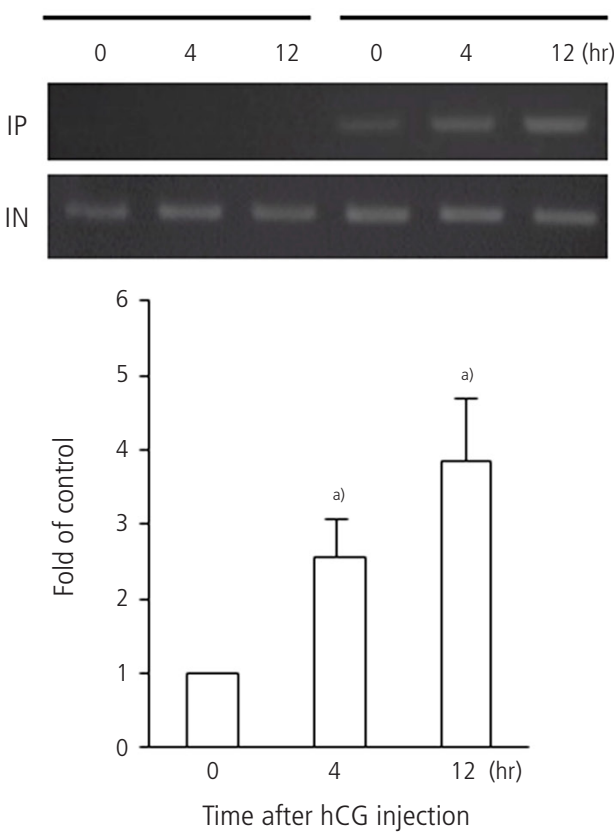

B

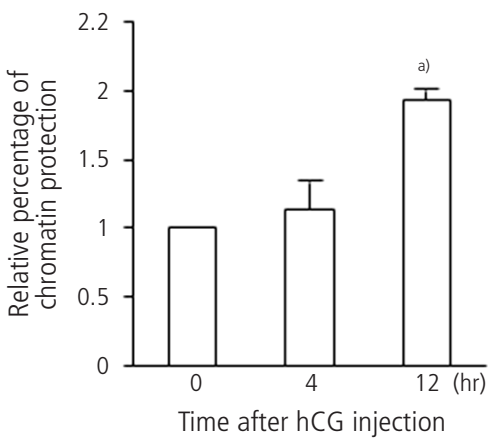

D P-CREB binding activities in the Cyp19a/ promoter
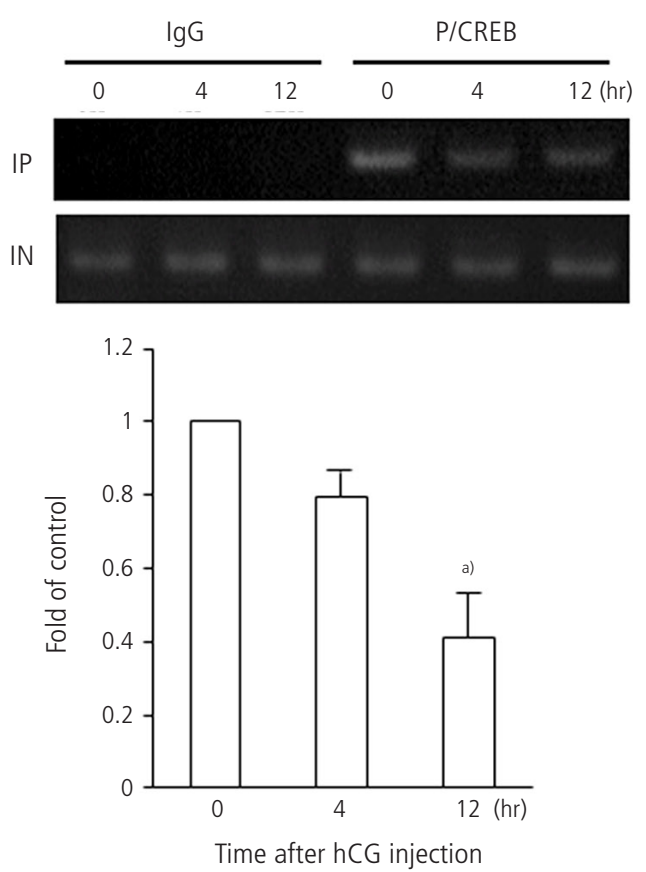

Fig. 5. Chromatin structure in the StAR and Cyp19a1 promoter regions and binding activities of CCAAT/enhancer binding protein (C/ EBP) $\beta$ to the StAR proximal promoter and phosphorylated CAMP-response element binding protein (P-CREB) to the Cyp19a1 promoter in granulosa cells undergoing luteinization during ovulation. To estimate the chromatin structure of the StAR promoter (A) and Cyp19a1 promoter (B), chromatin assay by real-time polymerase chain reaction with DNase I was performed in the proximal region 1 (-257 to -42 bp), proximal region 2 (-492 to -288 bp), and distal region (-1383 to -1195 bp) of the StAR promoter and in the Cyp19a1 promoter region (-169 to $-15 \mathrm{bp}$ ) in the granulosa cells obtained from rats treated with equine chorionic gonadotropin (eCG) before (0 hours), and 4 and 12 hours after human chorionic gonadotropin ( $\mathrm{hCG}$ ) injection. The relative percentage of chromatin protection against DNase I digestion was calculated, and data were expressed as a ratio of the value of 0 hours. High values indicate the condensed chromatin structure and low values indicate the open chromatin structure. C/EBP $\beta$ (C) and P-CREB (D) binding activities were analyzed by chromatin immunoprecipitation assay in the granulosa cells obtained from rats treated with eCG before (0 hours) and 4 hours, and 12 hours after hCG injection. Data were expressed as a percentage of the value of 0 hours. Values are means \pm SEM of three independent experiments and the ethidium bromide-stained gel are representatives of them. ${ }^{\text {al }} P<0.05$ versus 0 hours. The data were reported in the reference no. 15 .

$\beta$ is a crucial transcription factor that induces StAR gene expression in granulosa cells undergoing luteinization $[22,23]$. On the other hand, cAMP-response element binding protein
(CREB) up-regulates Cyp 19a1 gene expression in granulosa cells under FSH stimulation [24-26]. We investigated whether these transcription factors actually bind to or detach from the 


\section{Obstetrics \& Gynecology Science}

Norihiro Sugino. Molecular mechanisms of luteinization

promoter region of StAR and Cyp 19a 1 after hCG injection. Binding activities of C/EBP $\beta$ to the StAR proximal promoter region (proximal region 1) and of phosphorylated CREB to the Cyp19a1 promoter were analyzed by ChIP assay. Binding activities of C/EBP b to the StAR proximal promoter region increased after hCG injection (Fig. $5 \mathrm{C}$ ). In contrast, the binding activities of phosphorylated CREB to the Cyp19a1 promoter region decreased after hCG injection (Fig. 5D), suggesting the detachment of phosphorylated CREB from the Cyp19a1 promoter after hCG stimulation because the protein level of phosphorylated CREB increased after hCG injection (data not shown).

\section{Regulation of StAR gene expression by epigenetic mechanisms}

The DNA methylation status of the StAR proximal promoter region was unmethylated. Furthermore, hCG stimulation caused modifications of histones in the StAR proximal promoter region and loosened the chromatin structure, allowing the transcription factor, C/EBP $b$, to access its response element of the StAR proximal promoter. This mechanism contributes to the rapid induction of StAR gene expression in granulosa cells undergoing luteinization after ovulatory LH surge.

Christenson et al. [27] reported that the level of histone $\mathrm{H} 3$ acetylation of the StAR proximal promoter was increased in luteinized granulosa cells obtained after the induction of ovulation compared with the level in non-luteinized granulosa cells in the macaque. Hiroi et al. [28] also reported that the H3K9 methylation level of the StAR proximal promoter was immediately decreased in luteinizing granulosa cells after hCG stimulation. Our results are consistent with these reports.

The changes in chromatin structure and histone modifications in the StAR promoter were more apparent in the proximal promoter region than the distal promoter region. This finding is consistent with a recent report that histone acetylation levels are high at the region surrounding the transcription start site and are gradually less acetylated at increasing distances from the transcription start site [29]. Chromatin remodeling in the StAR promoter is spatiotemporally regulated by the different types of histone modifications in the granulosa cells undergoing luteinization after
hCG stimulation.

\section{Regulation of Cyp19a1 gene expression by epigenetic mechanisms}

Ovulatory stimulation also caused changes in histone modifications and chromatin structure in the Cyp19a1 promoter. hCG stimulation decreased levels of histone acetylation and H3K4me3 and increased levels of H3k27me3 in the Cyp19a 1 promoter region, which inactivate transcription. Thus, hCG stimulation made the chromatin structure of the Cyp19a 1 promoter region condensed through the alteration of histone modifications, allowing the transcription factor, phosphorylated CREB, to detach from its response element of the Cyp19a 1 promoter. The change in histone modifications and chromatin structure of the Cyp 19a 1 promoter are closely associated with the rapid suppression of Cyp19a1 gene expression in granulosa cells undergoing luteinization by ovulatory stimulation.

The signaling pathway of CAMP-protein kinase A-CREB is a key signal for Cyp19a1 gene expression in granulosa cells under FSH stimulation [24-26]. This pathway is also induced by the LH surge. However, it has been unclear about the mechanism by which Cyp 19a1 gene expression is suppressed in granulosa cells undergoing luteinization after the LH surge. This study showed the possible mechanism for the rapid suppression of Cyp19a1 gene expression in granulosa cells after ovulatory LH surge. The result that the binding activity of phosphorylated CREB to the Cyp19a1 promoter was decreased after hCG stimulation despite the constant protein expression of phosphorylated CREB in the nucleus strongly suggests the detachment of phosphorylated CREB from the Cyp 19a 1 promoter.

\section{Conclusion}

The present study showed that epigenetic changes (histone modifications and chromatin remodeling) occurred in the promoter regions of StAR and Cyp19a1 in granulosa cells undergoing luteinization during ovulation. Our results suggest that histone modifications and chromatin remodeling of the promoters are closely associated with the rapid changes of the expression of StAR and Cyp19a1 in luteinizing granulosa cells during ovulation, and provide a molecular mechanism for 


\section{Obstetrics \& Gynecology Science}

Vol. 57, No. 2, 2014

the shift from estrogen synthesis to progesterone synthesis in granulosa cells undergoing luteinization after the LH surge.

\section{Conflict of interest}

No potential conflict of interest relevant to this article was reported.

\section{Acknowledgments}

This work was supported in part by JSPS KAKENHI grants no. 23791846, 23890140, 24592471, 24791704, 25293343, 25462559, 25462560, and 25861495.

\section{References}

1. Ronen-Fuhrmann T, Timberg $R$, King SR, Hales KH, Hales $D B$, Stocco DM, et al. Spatio-temporal expression patterns of steroidogenic acute regulatory protein (StAR) during follicular development in the rat ovary. Endocrinology 1998;139:303-15.

2. Hickey GJ, Chen SA, Besman MJ, Shively JE, Hall PF, Gaddy-Kurten D, et al. Hormonal regulation, tissue distribution, and content of aromatase cytochrome P450 messenger ribonucleic acid and enzyme in rat ovarian follicles and corpora lutea: relationship to estradiol biosynthesis. Endocrinology 1988;122:1426-36.

3. Tanaka N, Espey LL, Kawano T, Okamura H. Comparison of inhibitory actions of indomethacin and epostane on ovulation in rats. Am J Physiol 1991;260(2 Pt 1):E170-4.

4. Lydon JP, DeMayo FJ, Funk CR, Mani SK, Hughes AR, Montgomery CA Jr, et al. Mice lacking progesterone receptor exhibit pleiotropic reproductive abnormalities. Genes Dev 1995;9:2266-78.

5. Tamura I, Sato S, Okada M, Tanabe M, Lee L, Maekawa $\mathrm{R}$, et al. Importance of C/EBP $\beta$ binding and histone acetylation status in the promoter regions for induction of IGFBP-1, PRL, and Mn-SOD by CAMP in human endometrial stromal cells. Endocrinology 2014;155:275-86.

6. Tamura I, Asada H, Maekawa R, Tanabe M, Lee L, Taketani T, et al. Induction of IGFBP-1 expression by CAMP is associated with histone acetylation status of the pro- moter region in human endometrial stromal cells. Endocrinology 2012;153:5612-21.

7. Tamura I, Taketani T, Lee L, Kizuka F, Taniguchi K, Maekawa $R$, et al. Differential effects of progesterone on COX-2 and Mn-SOD expressions are associated with histone acetylation status of the promoter region in human endometrial stromal cells. J Clin Endocrinol Metab 2011;96:E1073-82.

8. Yamagata Y, Asada H, Tamura I, Lee L, Maekawa R, Taniguchi $\mathrm{K}$, et al. DNA methyltransferase expression in the human endometrium: down-regulation by progesterone and estrogen. Hum Reprod 2009;24:1126-32.

9. Yamagata Y, Nishino K, Takaki E, Sato S, Maekawa R, Nakai $A$, et al. Genome-wide DNA methylation profiling in cultured eutopic and ectopic endometrial stromal cells. PLoS One 2014;9:e83612.

10. Sato S, Maekawa R, Yamagata Y, Asada H, Tamura I, Lee $L$, et al. Potential mechanisms of aberrant DNA hypomethylation on the $\mathrm{X}$ chromosome in uterine leiomyomas. J Reprod Dev 2013 Nov 29 [Epub]. DOI: 10.1262/ jrd.2013-095.

11. Maekawa R, Sato S, Yamagata Y, Asada H, Tamura I, Lee L, et al. Genome-wide DNA methylation analysis reveals a potential mechanism for the pathogenesis and development of uterine leiomyomas. PLoS One 2013;8:e66632.

12. Maekawa R, Yagi S, Ohgane J, Yamagata Y, Asada H, Tamura I, et al. Disease-dependent differently methylated regions (D-DMRs) of DNA are enriched on the $X$ chromosome in uterine leiomyoma. J Reprod Dev 2011;57:604-12.

13. Yamagata Y, Maekawa R, Asada H, Taketani T, Tamura I, Tamura $\mathrm{H}$, et al. Aberrant DNA methylation status in human uterine leiomyoma. Mol Hum Reprod 2009;15:25967.

14. Asada H, Yamagata Y, Taketani T, Matsuoka A, Tamura $\mathrm{H}$, Hattori $\mathrm{N}$, et al. Potential link between estrogen receptor-alpha gene hypomethylation and uterine fibroid formation. Mol Hum Reprod 2008;14:539-45.

15. Lee L, Asada H, Kizuka F, Tamura I, Maekawa R, Taketani $T$, et al. Changes in histone modification and DNA methylation of the StAR and Cyp19a1 promoter regions in granulosa cells undergoing luteinization during ovulation in rats. Endocrinology 2013;154:458-70.

16. Bird A. The essentials of DNA methylation. Cell 1992;70:5-8. 


\section{Obstetrics \& Gynecology Science}

Norihiro Sugino. Molecular mechanisms of luteinization

17. Keshet I, Lieman-Hurwitz J, Cedar H. DNA methylation affects the formation of active chromatin. Cell 1986;44:535-43.

18. Wu C. Chromatin remodeling and the control of gene expression. J Biol Chem 1997;272:28171-4.

19. Li B, Carey M, Workman JL. The role of chromatin during transcription. Cell 2007;128:707-19.

20. Fitzpatrick SL, Richards JS. cis-acting elements of the rat aromatase promoter required for cyclic adenosine $3^{\prime}, 5^{\prime}$-monophosphate induction in ovarian granulosa cells and constitutive expression in R2C Leydig cells. Mol Endocrinol 1993;7:341-54.

21. ilandre $D$, Delalande $C$, Durand $P$, Carreau S. Three promoters PII, PI.f, and PI.tr direct the expression of aromatase (cyp19) gene in male rat germ cells. J Mol Endocrinol 2007;39:169-81.

22. Fan HY, Liu Z, Johnson PF, Richards JS. CCAAT/enhancerbinding proteins (C/EBP)- $\alpha$ and $-\beta$ are essential for ovulation, luteinization, and the expression of key target genes. Mol Endocrinol 2011;25:253-68.

23. Yivgi-Ohana N, Sher N, Melamed-Book N, Eimerl S, Koler M, Manna PR, et al. Transcription of steroidogenic acute regulatory protein in the rodent ovary and placenta: alternative modes of cyclic adenosine 3', 5'-monophosphate dependent and independent regulation. Endocrinology 2009;150:977-89.

24. Fitzpatrick SL, Richards JS. Identification of a cyclic ad- enosine $3^{\prime}, 5^{\prime}$-monophosphate-response element in the rat aromatase promoter that is required for transcriptional activation in rat granulosa cells and R2C leydig cells. Mol Endocrinol 1994;8:1309-19.

25. Stocco C. Aromatase expression in the ovary: hormonal and molecular regulation. Steroids 2008;73:473-87.

26. Michael MD, Michael LF, Simpson ER. A CRE-like sequence that binds CREB and contributes to CAMPdependent regulation of the proximal promoter of the human aromatase P450 (CYP19) gene. Mol Cell Endocrinol 1997;134:147-56.

27. Christenson LK, Stouffer RL, Strauss JF 3rd. Quantitative analysis of the hormone-induced hyperacetylation of histone $\mathrm{H} 3$ associated with the steroidogenic acute regulatory protein gene promoter. J Biol Chem 2001;276:27392-9.

28. Hiroi $H$, Christenson LK, Chang L, Sammel MD, Berger SL, Strauss JF 3rd. Temporal and spatial changes in transcription factor binding and histone modifications at the steroidogenic acute regulatory protein (stAR) locus associated with stAR transcription. Mol Endocrinol 2004; 18:791-806.

29. Wang Z, Zang C, Rosenfeld JA, Schones DE, Barski A, Cuddapah $S$, et al. Combinatorial patterns of histone acetylations and methylations in the human genome. Nat Genet 2008;40:897-903. 